\title{
Morbidity, mortality and long term survival in patients with vascular resection in pancreatic cancer - single center experience
}

\author{
D. PINDAK, M. TOMAS*, J. DOLNIK, R. DUCHON, J. PAVLENDOVA \\ Department of Surgical Oncology of Slovac Medical University, National Cancer Institute Bratislava, Klenova 1, 833 10 Bratislava 3, Slovakia \\ *Correspondence: miroslav.tomas@nou.sk
}

Received September 1, 2016 / Accepted December 3, 2016

\begin{abstract}
Although venous resection in pancreatic cancer is widely used method, recently published data about its safety and survival benefit showed conflicting results. A retrospective case matched study was performed to compare the results of patients who underwent venous resection to those with no venous resection during radical surgery in pancreatic cancer.

From January 2010 to December 2015, 297 pancreatic resections due to pancreatic tumor were performed in the National Cancer Institute (NCI). Fifty-three patients with venous resection were identified and enrolled into the study and matched with 66 patients without vascular resection during radical resection of pancreatic head/body cancer. Both groups matched for age, ASA score, need for preoperative biliary drainage and clinical staging of the tumor. Morbidity was determined according Clavien and Dindo classification [1] and was similar in both groups of patients $(\mathrm{p}=0.48)$. Thirty day postoperative mortality was also equal in both groups 5.6 vs $4.5 \%(\mathrm{p}>0.99)$ and long term survival was with no significant difference. Median overall survival was 18.8 vs 20.7 months $(\mathrm{p}=0.33$ ) for patients with/without venous resection.

Therefore we consider venous resection in pancreatic cancer safe procedure with equal morbidity and perioperative mortality as in patients with no need for vascular resection and with the same long term survival if R0 resection is achieved.
\end{abstract}

Key words: pancreas, neoplasm, morbidity, mortality

Long term overall survival for pancreatic cancer is usually reported only $1-5 \%$ underlining poor prognosis of this type of cancer. For the majority of patients it is due to late diagnosis, as disease is very often diagnosed in advance stage. For those, diagnosed in earlier stages, where resection, as the only curative intervention can be applied, the 5 year survival is reported about $20-25 \%$ [2]. During last decades improvement in patient centralisation and intensive perioperative management yielded to decreased postoperative mortality which is today less than $5 \%$ in almost all centers worldwide. This allowed to expand the indications of extensive resections including vascular resections as an accepted intervention at least for venous resections $[3,4,6]$. Surprisingly, recent meta-analysis showed increased morbidity, mortality as well as worse long term survival [5]. Therefore we decided to evaluate our own results in these patients. Our study presents single center experience and results of concomitant venous resection in the treatment of pancreatic cancer in terms of morbidity and mortality.

\section{Patients and methods}

Patients. A retrospective case matched study was used to determine morbidity, 30 day mortality and long term survival in patients that underwent surgery for pancreatic tumor in tertiary cancer center, comparing results in those with and without venous resection. The study was approved by the institutional review board and a waiver of consent form was granted. Inclusion criteria: patients operated in the NCI Bratislava due to pancreatic tumor located in the head or body of the pancreas from January 2010 to December 2015 with the need for venous resection were included into the study. Subsequently we have identified patients with no need for venous resection operated in the same institution in the same time period matching in age, gender, American Society of Anesthesiologist (ASA) score, clinical staging (TNM) and tumor location (head/body of the pancreas) as a control group. Exclusion criteria: patients with concomitant arterial resection were excluded from the study. For long term survival analysis patients with no malignancy 
on final histology and with $\mathrm{R} 1$ resections and those with no adjuvant chemotherapy were excluded as well.

Data collection and statistics. Patient's medical records were used to identify those patients operated due to pancreatic tumor from 1.1.2010 to 31.12.2015 in NCI Bratislava, and information about age, ASA score, gender, preoperative clinical staging, computed tomography (CT)/magnetic resonance imaging (MRI) finding, need for preoperative biliary drainage, final histology, need for transfusion, antibiotic treatment, radiologic intervention, reoperation, date of the operation, operative time, length of ICU stay, total length of stay, information about adjuvant chemotherapy were identified and collected. Data cut-off for survival analysis was July 1,2016 . Postoperative morbidity was determined according Clavien and Dindo classification [1].

Statistical analysis. Statistical relationships were evaluated by $t$ test, Fisher exact test and Fisher-Freeman-Halton test. For testing differences in Clavien and Dindo classification we used non parametric Mann-Whitney U test and KolmogorovSmirnov test. Kaplan Meier's method was used for long term survival analysis and Log-rank test to evaluate differences between different groups of patients. All statistical hypothesis were tested on significance level alpha $=0.05$. Software Statsdirect version 3.0.177 was used.

Surgical technique. Standard Whipple procedure was performed in the majority of patients, with conventional heparin at the dose of 100 units per kilogram, used during clamping of mesenterico-portal veins, with subsequent continual anticoagulation treatment only in those patients with prosthetic reconstruction. For the patients with total pancreatectomy with the need for interruption of left gastric vein a standard subtotal gastrectomy was added to ensure good venous drainage of remained stomach.

\section{Results}

From January 2010 to December 2015297 pancreatic resections were performed in our institution. Fifty-three patients with venous resection during pancreatic resection (intervention group) due to tumor located in the pancreatic head/body were identified and other 66 patients matching with them in

Table 1. Group's characteristics showing no significant difference between the groups

\begin{tabular}{lccc}
\hline & $\begin{array}{c}\text { Venous } \\
\text { resection } \\
\mathbf{n}=\mathbf{5 3}\end{array}$ & $\begin{array}{c}\text { No venous } \\
\text { resection } \\
\mathbf{n}=\mathbf{6 6}\end{array}$ & p value \\
\hline Age (mean) & 61.4 & 61.6 & $0.95^{1}$ \\
Gender (male/female \%) & $54.7 / 45.3$ & $60.6 / 39.4$ & $0.59^{2}$ \\
ASA classification grade 1/2/3/4 (n) & $2 / 36 / 15 / 0$ & $7 / 37 / 22 / 0$ & $0.14^{3}$ \\
Tumor size (mean in mm) & 38.8 & 41.0 & $0.59^{1}$ \\
Preoperative biliary stent $(\mathrm{n} / \%)$ & $31 / 58.5$ & $36 / 54.5$ & $0.86^{4}$ \\
\hline${ }^{1}$ t test, ${ }^{2}$ Fisher's exact test, ${ }^{3}$ Fisher-Freeman-Halton test, ${ }^{4}$ Mann-Whitney \\
U test
\end{tabular}

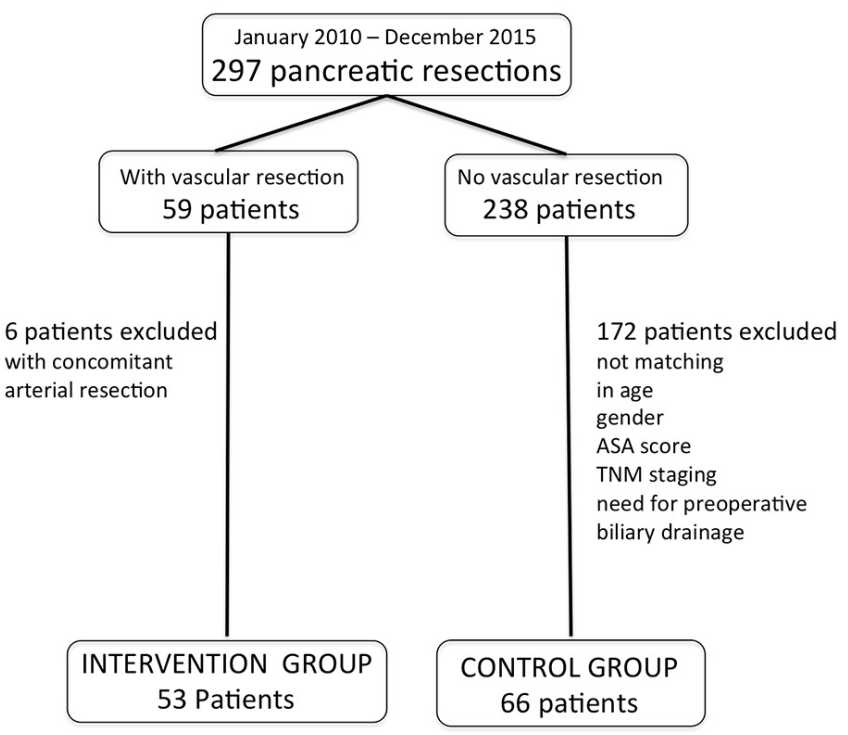

Figure 1. Patient selection

age, ASA score, need for preoperative biliary drainage and clinical staging were identified as a control group (Figure 1).

The mean age was 61.4 and 61.6 years $(\mathrm{p}=0.95)$, ASA score showed non significant difference as well $(\mathrm{p}=0.14)$. Most common clinical stage T2N0 with mean tumor size 38.8 vs 41 millimeters $(\mathrm{p}=0.59)$ was detected in both groups according preoperative CT and MRI. Male/female ratio was also equal $29 / 24$ versus $40 / 26(p=0.59)$. Segmental venous resection was performed in 41 patient with prosthetic graft reconstruction in $27(50.1 \%)$, wedge resection with venorrhaphy was done in 12 patients. All groups characteristics are shown in the Table 1.

30 days mortality. There were 3 deaths in both groups. Two severe sepsis as a cause in each group where the septic focus was not identified, one necrotising pancreatitis and one myocardial infarction were detected as causes. Statistical analysis found no significant difference between the groups ( $p>0.99$ ).

Postoperative morbidity. Postoperative morbidity was calculated according Clavien and Dindo classification for both groups of patients and is shown in Table 2. Statistical

Table 2. Morbidity according Clavien and Dindo Classification showed no significant difference between the groups

\begin{tabular}{cccc}
\hline $\begin{array}{c}\text { Clavien and } \\
\text { Dindo }\end{array}$ & $\begin{array}{c}\text { Venous resection } \\
\mathbf{n}=\mathbf{5 3}(\mathbf{n} / \%)\end{array}$ & $\begin{array}{c}\text { No venous resection } \\
\mathbf{n}=\mathbf{6 6}(\mathbf{n} / \%)\end{array}$ & p value \\
\hline 1 & $17 / 32.1$ & $19 / 28.8$ & \\
2 & $24 / 45.3$ & $31 / 47.0$ & \\
$3 \mathrm{a}$ & $3 / 5.7$ & $7 / 10.6$ & $0.48^{1}$ \\
$3 \mathrm{~b}$ & $5 / 9.4$ & $5 / 7.6$ & \\
$4 \mathrm{a}$ & $1 / 1.9$ & $1 / 1.5$ & \\
$4 \mathrm{~b}$ & $0 / 0$ & $0 / 0$ & \\
5 & $3 / 5.6$ & $3 / 4.5$ & \\
\hline
\end{tabular}

${ }^{1}$ Kolmogorov-Smirnov test 


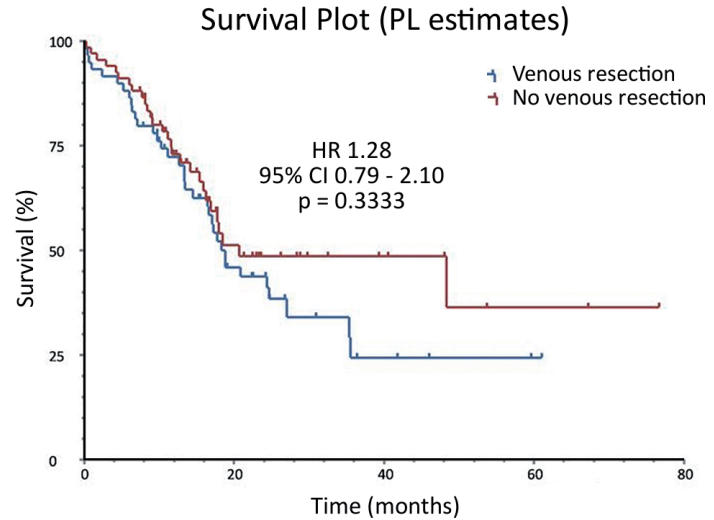

Figure 2. Overall survival showed no significant difference between the groups, censored observations are indicated on the Kaplan-Meier curve as tick marks.

analysis showed no significant differences between groups $(\mathrm{p}=0.48)$.

Long term survival. There were two non $\mathrm{R} 0$ resections in both groups, so for the long term survival analysis data from 51 and 64 patients were used, as all patients were able to at least start the adjuvant chemotherapy.

The median follow-up was 14.7 months and median survival for patients with venous resection was 18.8 months comparing to 20.7 months for those with no venous resection ( $\mathrm{p}=0.33$ ) Survival analysis by Kaplan Meier method is showed in Figure 2. with no significant difference calculated by Log rank test -hazard ratio 1.28 95\%CI (0.77-2.09) $\mathrm{p}=$ 0.33 . One - year survival rates and estimated 3 - and 5 - year survival rates are shown in the Table 3.

\section{Discussion}

Venous resections in the treatment of pancreatic cancer are well established in the majority of centers performing pancreatic resections, despite the fact, that the quality of data supporting its use is limited. There are 3 large meta-analyses published recently $[3,4,5]$ based on retrospective cohort studies and retrospective observational studies. There is no

Table 3. One-, 3-, and 5 year survival

\begin{tabular}{lcc}
\hline & $\begin{array}{c}\text { Venous resection } \\
\mathbf{n}=\mathbf{5 1}\end{array}$ & $\begin{array}{c}\text { No venous resection } \\
\mathbf{n}=\mathbf{6 4}\end{array}$ \\
\hline Number of survivors & 28 & 31 \\
1 year survival (\%) & 72.3 & 72.9 \\
3-year survival estimated (\%) & 24.4 & 48.6 \\
95\% CI & $10.9-40.6$ & $33.6-62.1$ \\
5-year survival estimated (\%) & 24.4 & 36.4 \\
95\% CI & $10.9-40.6$ & $14.9-58.5$ \\
\hline
\end{tabular}

$\mathrm{CI}$ - confidence interval one randomized study to date, and probably never will be, due to ethical problems.

The first two mentioned meta-analyses published in 2012 and 2014 [3, 4] confirmed comparable morbidity, mortality and long term survival (despite selection bias). Recently published paper from F. Giovinazzo and coauthors [5] , however, showed in their meta-analysis of 27 studies involving a total of 9005 patients increased postoperative mortality (risk difference (RD) $0.01,95 \mathrm{CI} 0.00$ to $0.03, \mathrm{p}=0.02$ ), higher rates of non-radical surgeries (RD 0.09, CI $0.06-0.13, \mathrm{p}<0.001$ ) and worse survival (6 studies, 1935 patients) after resections with portal - superior mesenteric veins (PV-SMV) resection. One-, 3- and 5- year survival were worse in the PV- SMV resection group: $\mathrm{HR} 1.23$ (95\% CI $1.07-1.43, \mathrm{p}=0.005)$, $1.48(1.14-1.91, \mathrm{p}=0.004)$ and $3.18(1.95-5.19, \mathrm{p}<0.001)$. Authors assume, that the explanation could be related to more advanced disease in the group of patient with PV-SMV resections, as used TNM classification system does not consider PV - SMV infiltration, despite its negative prognostic impact on survival $[7,8]$. This suggest that, even though tumours with and without PV-SMV invasion are classified within the same TNM stage, the biology of this two categories may be different. Moreover authors did not consider the use of neoadjuvant and adjuvant treatment.

The major advantage of our study is case matched design, with well balanced study groups, with the use of standard adjuvant chemotherapy in all patients and exclusion of all patients with $\mathrm{R} 1 / 2$ resections. Limitations are small sample size and impossibility to consider histologically confirmed venous invasion, as our final histopathological reports did not provided this information in all patients.

According to our experience, in accordance with some recently published single center studies $[9,10]$ the postoperative morbidity, mortality and long term survival is equal in patients with/without venous resections in the treatment of localized pancreatic cancer. Moreover the long term survival for the selected patient with PV-SMV resections after R0 resection followed with subsequent adjuvant chemotherapy is almost the same. The only $\mathrm{R} 1$ resections in our study was due to mistakes in evaluating operative frozen section analysis, and usually at the AMS margin, not at the venous margins. Despite this fact we suggest to use at least $10 \mathrm{~mm}$ margins on the vein, if possible. Probably it is the reason for prosthetic graft reconstruction as the most common technique used in our patients $(50.1 \%)$.

Despite the results of last mentioned meta-analysis [5] reporting worse results in PV-SMV resections in terms of morbidity, mortality and survival, we still suggest to perform PV-SMV resections as a standard approach. However, our findings from a retrospective single-center study will ideally need confirmation in multicenter studies.

Conclusion. According our experience we consider venous resection in pancreatic cancer safe procedure with the same morbidity and postoperative mortality as in those patients with no need for venous resection. The long term survival 
if $\mathrm{R} 0$ resection is achieved and patient will receive adjuvant chemotherapy is also equal.

\section{References}

[1] DEOLIVEIRA ML, WINTER JM, SCHAFER M, CUNNINGHAM SC, CAMERON JL et al. Assessment of Complications After Pancreatic Surgery. Ann Surg 2006; 244: 931-937. https://doi.org/10.1097/01.sla.0000246856.03918.9a

[2] HACKERT T, BUCHLER MW. Pancreatic cancer: advances in treatment, results and limitations. Dig Dis 2013; 31: 51-56. https://doi.org/10.1159/000347178

[3] ZHOU Y, ZHANG Z, LIU Y, LI B, XU D. Pancreatectomy combined with superior mesenteric vein-portal vein resection for pancreatic cancer: a meta-analysis. World J Surg 2012; 36: 884-891. https://doi.org/10.1007/s00268-012$\underline{1461-\mathrm{Z}}$

[4] YU XZ, LI J, FU D L, DI Y, YANG F et al. Benefit from synchronous portal-superior mesenteric vein resection during pancreaticoduodenectomy for cancer: A meta-analysis. Eur J Surg Oncol 2014; 40: 371-378. https://doi.org/10.1016/j. ejso.2014.01.010

[5] GIOVINAZZO F, TURRI G, KATZ M H, HEATON N, AHMED I. Meta-analysis of benefits of portal-superior mesenteric vein resection in pancreatic resection for ductal adenocarcinoma. Br J Surg 2016; 103: 179-191. https://doi. org/10.1002/bjs.9969

[6] HACKERT T, SCHNEIDER L, BUCHLER MW. Current state of vascular resections in pancreatic cancer surgery. Gastroenterol Res Pract 2015; 2015: 120207 https://doi. org/10.1155/2015/120207

[7] FUKUDA S, OUSSOULTZOGLOU E, BACHELLIER P, ROSSO E, NAKANO $\mathrm{H}$ et al. Significance of the depth of portal vein wall invasion after curative resection for pancreatic adenocarcinoma. Arch Surg 2007; 142: 172- 179. https://doi. org/10.1001/archsurg.142.2.172

[8] SUENAGA M, FUJII T, KANDA M, TAKAMI H, OKUMURA $N$ et al. Pattern of first recurrent lesions in pancreatic cancer: hepatic relapse is associated with dismal prognosis and portal vein invasion. Hepatogastroenterology 2014; 61: $1756-1761$.

[9] FLIS V, POTRC S, KOBILICA N, IVANECZ A. Pancreaticoduodenectomy for ductal adenocarcinoma of the pancreatic head with venous resection. Radiol Oncol 2016; 50: 321-328. https://doi.org/10.1515/raon-2015-0017

[10] MARSONER K, LANGEDER R, CSENGERI D, SODECK G, MISCHINGER HJ et al. Portal vein resection in advanced pancreatic adenocarcinoma: is it worth the risk? Wien Klin Wochenschr 2016; 128: 566-572. https://doi.org/10.1007/ s00508-016-1024-7 